Nuclear GAPDH signaling mediates pathological cardiac hypertrophy

Manling Zhang ${ }^{1, *}$, Taro Kariya ${ }^{1,{ }^{*}}$, Genri Numata ${ }^{2,{ }^{*}}$, Adrianan Ramos ${ }^{3 *}$, Hideyuki Sasaki ${ }^{1,{ }^{*}}$,

Masaki Iwakiri ${ }^{4}{ }^{*}$, Masayuki Sasaki ${ }^{1}$, Norimichi Koitabashi ${ }^{1}$, Guangshuo Zhu ${ }^{1}$, Tsuyoshi

Tsujimura $^{3}$, Dong-ik Lee ${ }^{1}$, Carlos Tristan $^{3}$, Neelam Shahani ${ }^{3}$, Yukihiro Tsuchiya ${ }^{5}$, Hanna Jaaro-

Peled $^{3}$, Barbara Slusher ${ }^{6,7}$, David A. Kass ${ }^{1}$, Kyoji Taguchi $^{8}$, Yoshie Horiguchi ${ }^{9}$, Toshiaki

Saitoh $^{10}$, Koko Ishizuka ${ }^{3}$, Akira Sawa ${ }^{3, \#}$, and Eiki Takimoto ${ }^{1, \#}$

${ }^{1}$ Division of Cardiology, Departments of ${ }^{3}$ Psychiatry, ${ }^{6}$ Neurology, and ${ }^{7}$ Brain Science Institute, Johns Hopkins University School of Medicine

Departments of ${ }^{2}$ Cardiovascular Medicine and ${ }^{4}$ Anesthesiology, University of Tokyo

Departments of ${ }^{5}$ Pharmacology, ${ }^{8}$ Medicinal Pharmacology, and ${ }^{9}$ Medicinal Chemistry

Showa Pharmaceutical University

${ }^{10}$ Department of Pharmacy, Faculty of Pharmaceutical Sciences, Aomori University

*These six authors contributed equally to this work

${ }^{\#}$ Corresponding authors: etakimo1@jhmi.edu, asawa1@jhmi.edu 


\title{
One-sentence summary:
}

This study shows a novel function of GAPDH in homeostatic control of the heart, which is disturbed and results in cardiac hypertrophy with pathological stressors.

\begin{abstract}
:
Pathological stressors disrupt cellular and organ homeostasis, causing various diseases. We discovered a novel role for glyceraldehyde-3-phosphate dehydrogenase (GAPDH) in the pathological growth response of the heart, independent of its functions in glycolysis and cell death. In a cellular model for cardiac hypertrophy, endothelin-1 elicited nuclear translocation of GAPDH and activation of p300 histone acetyl-transferase (HAT), followed by activation of myocyte enhancer factor 2 (MEF2). GAPDH nuclear translocation and p300 HAT activation was also identified in rodent pathological hypertrophied hearts. The hypertrophy was markedly ameliorated by molecular and pharmacological interventions that antagonize the nuclear GAPDH pathway, including a novel antagonist selective to its nuclear function. This pathway may be the key to stress response/homeostatic control, and thus the potential therapeutic target for stressassociated diseases.
\end{abstract}




\section{Main Text:}

Besides glycolytic function, glyceraldehyde-3-phosphate dehydrogenase (GAPDH) translocate to the nuclei in response to stress where it has been described to mainly regulate cell death (1-4). The nuclear GAPDH pathway is triggered by a specific oxidation/S-nitrosylation of GAPDH at cysteine-150, which enables the interaction of the pool of GAPDH with Siah1 $(5,6)$. The protein complex translocates to the nucleus where GAPDH can affect p300, p53 and p53 upregulated modulator of apoptosis (PUMA) resulting in cell death; in this cascade, only a small fraction of GAPDH is converted to a signaling molecule, and the overall change in cytosolic and glycolytic GAPDH is negligible (5, 7). Despite it is involvement in cell death, it remains unclear whether and how this cascade plays general and diverse roles.

The heart is among the organs with the highest expression of GAPDH (8), which has been simply thought to play a "house keeping" glycolytic role in this organ. The heart develops hypertrophy or abnormal growth in response to pathological stress, which can ultimately result in the organ failure (9). Indeed, cardiac hypertrophy and failure is a leading cause of death worldwide, imposing an enormous burden on society (10). Although p300 has been implicated in this pathophysiology $(11,12)$, the overall significance and its regulatory mechanism remain elusive. We hypothesize that nuclear GAPDH-p300 signaling may mediate this stress response, which disrupts critical homeostasis of cardiac myocytes and causes hypertrophic growth.

To address this question, we used a mouse model of pressure-overload cardiac hypertrophy induced by transverse aortic constriction (TAC) (13). In this model, cardiac hypertrophy is developed at 7 to 10 days after TAC, transitioning to failure in 63 days after TAC. The histone acetyl transferase (HAT) activity of p300 was markedly increased in TAC hearts at 10 days after the constriction (TAC 10D), and remained high at 63 days (TAC 63D) (Fig. 1A), 
which was associated with nuclear accumulation of GAPDH in cardiac myocytes isolated from TAC hearts, as detected by immunostaining (Fig. 1B). Nuclear GAPDH accumulation was confirmed by protein sub-cellular fractionation analysis in the heart tissues (Fig. 1C). GAPDH in the nuclear fraction was increased in both TAC 10D and TAC 63D hearts, but not in sham hearts, while GAPDH in the cytosol fraction was unaltered under all conditions. These results are consistent with our hypothesis that TAC elicits nuclear translocation of GAPDH followed by activation of p300. PUMA was unchanged in TAC 10D hearts (fig. S1), indicating that the mechanism involving nuclear GAPDH in the heart is different from previously described (ref).

To test this possibility, we needed to block the nuclear GAPDH cascade. We previously reported that deprenyl, a monoamie oxidase (MAO) inhibitor, and its structural analogue could selectively block the nuclear cascade in cells by inhibiting GAPDH-Siah1 protein interaction (14). We performed extended screening of structural analogues of deprenyl, and identified more potent and selective blockers of GAPDH-Siah1 binding (data not shown). One of the most promising compounds was (1R, 3R)-1, 3-dimethyl-2-propargyl-1, 2, 3, 4-tetrahydroisoquinoline (designated RR in the present manuscript) (Fig. 2A and Supplementary Information). While deprenly's MAO inhibitory action could elicit adverse cardiovascular effects due to excess levels of catecholamine and serotonin $(15,16), \mathrm{RR}$ did not inhibit MAO-A/B at a wide range of concentrations in cardiac myocytes (Fig. 2B). Moreover, RR did not affect GAPDH glycolytic activity in cardiac myocytes at $1 \mathrm{nM}$ (Fig. 2C), the dose used in our subsequent studies. Comprehensive in vitro screening of off-target activities revealed no significant interaction of RR with receptors, transporters and enzymes at the concentration of $1 \mu \mathrm{M}$, which is 1,000 fold high in comparison to that at which GAPDH-Siah1 binding was inhibited (Table S1). These results support the high specificity of RR in inhibiting the GAPDH-Siah1 binding. 
We tested the role of nuclear GAPDH pathway in cardiac cellular hypertrophy model induced by Gq signal stimulation, a central trigger for pathological hypertrophy response and oxidative stress (17-21). Stimulation of cardiac myocytes with a Gq agonist endothelin 1 (ET1) $(0.05 \mu \mathrm{M}, 48 \mathrm{~h})$ augmented GAPDH-Siah1 binding, which was normalized by co-treatment with RR at $1 \mathrm{nM}$ (Fig. 3A). Both immunofluorescent cell staining and biochemical fractionation indicated that nuclear translocation of GAPDH occurred in response to ET1, and that RR blocked the translocation (Fig. 3, B and C, and fig. S2A). Consistent with the ET1-elicited translocation, accumulation of sulfonated GAPDH (reflecting oxidized nuclear GAPDH (5)) was observed in the nucleus, which was also blocked by RR (fig. S2, B and C). Augmented p300 activity leads to the activation of cardiac hypertrophic gene program by increase in the transcriptional activity of myocyte enhancer factor 2 (MEF2) (22-24). ET1 increased the levels of p300 acetylation (reflecting p300 HAT activity (25)) and the activity of MEF2 (Fig. 3D), and induced robust cellular hypertrophic response including increases in cell surface area, protein synthesis assayed by $\left[{ }^{3} \mathrm{H}\right]$ leucine uptake, and B-type natriuretic peptide (BNP) expression (Fig. 3E). Of note, under this condition, PUMA mRNA expression was unchanged (fig. S2D). Importantly, all of these changes were attenuated by RR (Fig. 3, D and E). These results provide pharmacological evidence that the GAPDH-p300-MEF2 cascade, which is independent of the PUMA-mediated death signaling, plays a crucial role in cardiac hypertrophic response.

To determine the significance of the nuclear GAPDH cascade in vivo and explore a novel therapeutic strategy for cardiac hypertrophy, we tested the RR compound in the pressure overload (TAC) model (see Fig. 1). Daily treatment with RR (0.25 mg/kg/day i.p.), initiated at the induction of TAC, markedly reduced the levels of nuclear GAPDH and p300 HAT activity in TAC 10D hearts (Fig. 3F), and ameliorated cardiac hypertrophy remodeling in vivo, as assessed 
by heart weight, myocyte cell size (cell surface area of cardiac myocytes), fibrosis (Fig. 3, G and

H) and pathological gene expression profiles (fig. S3, A and B). RR treatment also improved TAC-induced cardiac functional impairment (reduction in FS, fractional shortening) and chamber enlargement (increase in LV-EDD, left ventricular end-diastolic dimension), assessed by echocardiography (Fig. 3I). An invasive hemodynamic study using pressure volume loop analyses further revealed improvement of cardiac systolic (dPdtmax) and diastolic (Tau) performance with RR treatment despite sustained pressure-overload (Fig. 3J and fig. S3C). These results indicate that the RR compound is effective in blocking the nuclear GAPDH cascade and consequent changes in cardiac hypertrophy/remodeling and function in vivo.

In most clinical settings, treatment is started only after pathological changes have occurred. Thus, we next tested the efficacy of RR treatment in pre-existing cardiac hypertrophy. Hearts exposed to pressure-overload for 7 days (TAC 7D) developed hypertrophy with 50\% increase in left ventricular (LV) mass, compared with sham controls, by echocardiography (Fig. 3K). Two weeks of RR treatment to TAC 7D hearts significantly inhibited further increase in LV mass, compared with vehicle treatment (Fig. 3K and fig. S3D), and resulted in smaller cardiac myocytes (Fig. 3K and fig. S3D) and improved BNP expression profile (fig. S3D).

We further validated this mechanism employing molecular intervention both in vitro and in vivo. The human lysine residue K227 of GAPDH is crucial for its binding to Siah1 (corresponding to mouse K225A mutant). Expression of mutant GAPDH with substitution of this lysine interferes with endogenous GAPDH binding with Siah1 to function as a dominantnegative in this cascade (5). Thus, we first confirmed that this dominant-negative human GAPDH-K227 remained in the cytoplasm in cultured cardiac myocytes exposed to ET1 (Fig. 4A). Consistent with the pharmacological intervention, expression of this dominant-negative 
GAPDH blocked the augmentation of MEF2 activity (Fig. 4B), and outcome measures for the cardiac hypertrophic response, including an increase in BNP expression and cell surface area (Fig. 4C, and fig. S2E). Next, we generated a cardiac myocyte-specific knock-in mouse harboring GAPDH K225A mutant (Fig. 4D), using the tamoxifen-inducible Cre-loxP system, and examined the hypertrophic response of the heart to pressure overload (TAC). K225A mutant hearts revealed virtually abrogated GAPDH-Siah1 binding after exposure to10-day TAC (Fig.4E). Importantly, this was associated with ameliorated cardiac function, as assessed by echocardiographic measures of FS (Fig. 4F), indicating the response of K225A mutants as better adaptation vs wild types [note, the cardiac function of wild types and K225A mutants at the baseline were indistinguishable (fig. S4)]. Therefore, inhibition of nuclear GAPDH cascade could ameliorate pathological hypertrophic remodeling consistently by pharmacological or by molecular intervention.

In the present study, we demonstrate a pivotal role for the nuclear GAPDH cascade in cardiac hypertrophy in response to pathological stress in vitro and in vivo. We propose that GAPDH may be a key homeostatic mediator in living organisms, given its robust expression in many tissues/organs. Interestingly, the molecular mechanism elicited by nuclear translocation of GAPDH in the heart is distinct from the cascades reported in the brain, implying the general and tissue/context-specific roles of GAPDH in stress response. It would be very interesting to identify the roles for nuclear GAPDH pathway in other organs and under diverse stress situations. Our novel compound that specifically and potently blocks the nuclear GAPDH cascade, not only provides a potent means to determine these processes, but also open a window for potential therapeutic avenue. Since this cascade is likely to be involved in a variety of pathological conditions in which organ homeostasis is disturbed, it may have broad clinical applicability. 


\section{References and Notes:}

1. D. M. Chuang, C. Hough, V. V. Senatorov, Annu. Rev. Pharmacol. Toxicol. 45, 269 (2005).

2. M. A. Sirover, Biochim. Biophys. Acta 1810, 741 (2011).

3. D. Zala et al., Cell 152, 479 (2013).

4. C. Tristan, N. Shahani, T. W. Sedlak, A. Sawa, Cell. Signal. 23, 317 (2011).

5. M. R. Hara et al., Nat. Cell Biol. 7, 665 (2005).

6. M. R. Hara, M. B. Cascio, A. Sawa, Biochim. Biophys. Acta 1762, 502 (2006).

7. N. Sen et al., Nat. Cell Biol. 10, 866 (2008).

8. T. Brattelid et al., BMC Mol. Biol. 11, 22 (2010).

9. J. A. Hill, E. N. Olson, N. Engl. J. Med. 358, 1370 (2008).

10. S. Neubauer, N. Engl. J. Med. 356, 1140 (2007).

11. R. J. Gusterson, E. Jazrawi, I. M. Adcock, D. S. Latchman, J. Biol. Chem. 278, 6838 (2003).

12. T. Yanazume, T. Morimoto, H. Wada, T. Kawamura, K. Hasegawa, Mol. Cell. Biochem. 248, 115 (2003).

13. E. Takimoto et al., Nat.Med. 11, 214 (2005).

14. M. R. Hara et al., Proc. Natl. Acad. Sci. U. S. A. 103, 3887 (2006).

15. M. Wimbiscus, O. Kostenko, D. Malone, Cleve. Clin. J. Med. 77, 859 (2010).

16. M. R. Bristow, Circ. Res. 109, 1176 (2011).

17. E. Takimoto, D. A. Kass, Hypertension 49, 241 (2007).

18. T. Ago et al., Cell 133, 978 (2008).

19. E. Takimoto et al., J. Clin. Invest. 119, 408 (2009). 
20. N. Wettschureck et al., Nat. Med. 7, 1236 (2001).

21. G. W. Dorn, 2nd, T. Force, J. Clin. Invest. 115, 527 (2005).

22. Y. Kim et al., J. Clin. Invest. 118, 124 (2008).

23. T. I. Slepak et al., J. Biol. Chem. 276, 7575 (2001).

24. J. Q. Wei et al., Circulation 118, 934 (2008).

25. P. R. Thompson et al., Nat. Struct. Mol. Biol. 11, 308 (2004).

\section{Acknowledgements:}

We thank Dr. Pamela Talalay for critical reading of the manuscript. We also thank Ms. Yukiko Lema for organizing the figures and manuscript. This work was supported by USPHS grants of HL-093432 (E.T.), MH-094268 Silvo O. Conte center (A.S.), HL-077180 (D.A.K.), HL-059408 (D.A.K.), HL-07227 (M.Z.), MH-107730 (A.S.), MH-105660 (A.S. and K.I.), F31NS070459

(C.T.), and grants from AHA (GIA 7700071) (E.T.), AHA post-doctoral fellowship (M.Z.), Stanley (A.S.), RUSK (A.S.), S-R foundations (A.S.), and NARSAD (A.S. and K.I.). 


\section{Fig. 1. Augmented p300 HAT activity and nuclear accumulation of GAPDH in}

\section{hypertrophied hearts exposed to chronic pressure-overload (TAC).}

A. P300-HAT activity in TAC hearts ( $\mathrm{n}=4$ in each group). B. Representative pictures showing gross anatomy of TAC hearts (upper panels) and immunofluorescent staining for GAPDH in cardiac myocytes isolated from TAC hearts (middle and lower panels). Scale bars, $5 \mathrm{~mm}$ (upper panel) and $10 \mu \mathrm{m}$ (lower panel). C. Western blots (upper panels) and quantification (bar graphs, $\mathrm{n}=6$ in each group) with fractionated proteins from TAC hearts. TAC 10D, outcome of TAC for 10 days; and TAC 63D, outcome of TAC for 63 days. Error bar represents mean \pm SEM. *p $<$ 0.05 by one-way ANOVA with Tukey's multiple comparisons test.

Fig. 2. A novel compound that blocks GAPDH-Siah1 binding and the nuclear GAPDH cascade.

A. Chemical structure of "(1R, 3R)-1, 3-dimethyl-2-propargyl-1, 2, 3, 4-tetrahydroisoquinoline (RR compound)" in comparison to deprenyl. B. In vitro MAO activity assay. C. Glycolytic activity in cultured rat neonatal cardiac cells exposed to $1 \mathrm{nM} R \mathrm{R}$ for $48 \mathrm{~h}$.

Fig. 3. Mechanism of the nuclear GAPDH cascade in cardiac cellular hypertrophy and requirement of such cascade in cardiac hypertrophy/remodeling in vivo, which are validated by a specific blocker of GAPDH-Siah1 binding (RR)

A. Cell lysates (cardiac myocytes exposed to ET1 in the presence or absence of RR compound) immunoprecipitated with Siah1 antibody and probed for GAPDH (upper panel) and the quantification (lower panel, results from 4 experiments). B. Representative immune-fluorescent staining for GAPDH (quantification in fig. S2a). Scale bar, $50 \mu \mathrm{m}$. C. Quantification of Western 
blots for GAPDH in the nuclear fraction of cardiac myocytes exposed to ET1 in the presence or absence of RR compound. D. Quantification of p300 acetylation and MEF2 activity. E. Assessment of cardiac myocyte hypertrophy by cell surface area (left), protein synthesis (middle), and BNP gene expression $(N p p b)$. F. Quantification of Western blots for GAPDH in nuclear fraction (left) and p300 HAT activity in TAC hearts with vehicle (Veh) or RR (RR) treatment ( $n=3-4$ in each group). G. Representative cross-sections of hearts (upper panel) and histology (middle panels, Masson Trichrome Staining; lower panels, WGA staining). Scale bars, $5 \mathrm{~mm}$ (upper panel), $100 \mu \mathrm{m}$ (middle panel) and $10 \mu \mathrm{m}$ (lower panel). H. Assessment of cardiac hypertrophy: heart weight normalized by tibia length (left), average cross-sectional area (CSA) of cardiac myocytes (middle) and \% fibrosis (right). $n=5-7$ in each group. I. Echocardiogram: representative M-mode image (left), fractional shortening (FS) (middle), and left ventricular chamber size at end-diastole (LV-EDD) (right). $\mathrm{n}=5-7$ in each group. J. Comprehensive cardiac functional assessment from invasive pressure-volume loop analysis: representative loops during preload reduction (left), contractile parameter (dPdtmax) (middle), and relaxation parameter (Tau) (right). $\mathrm{n}=5-7$ in each group. K. RR treatment in pre-existing cardiac hypertrophy. Left ventricular mass calculated from echo-cardiogram before [pre-treatment at 7 days after the aortic constriction started (TAC 7D)] and after [post-treatment at 3 weeks after the constriction started (TAC 21D)] treatment (left). $n=5-7$ in each group. Left ventricular mass increase over 2 weeks ( $\Delta \mathrm{LV}$ mass increase) with vehicle or RR treatment (middle), and terminal myocyte size by crosssectional area (CSA) analysis (right). Error bar represents mean \pm SEM. ${ }^{*} \mathrm{p}<0.05$ by one-way ANOVA with Tukey’s multiple comparisons test or unpaired two tailed t test in panel K.. 
Fig. 4. Mechanism of the nuclear GAPDH cascade in cardiac cellular hypertrophy and requirement of such cascade in cardiac hypertrophy/remodeling in vivo, which are validated by a cardiac myocyte-specific knock-in mouse model (GAPDH K225A mutant).

A. Immuno-fluorescent staining for exogenous GAPDH (HA-tagged) in cardiac cells adenovirally transfected with wild type GAPDH (Ad-GAPDH-WT) or mutant GAPDH (Ad-GAPDHK227) under ET1 stimulation $(0.05 \mu \mathrm{M}, 48 \mathrm{~h})$. Scale bar, $50 \mu \mathrm{m}$. B. MEF2 activity in cardiac cells transfected with wild type GAPDH (Ad-GAPDH-WT) or mutant GAPDH (Ad-GAPDHK227) and stimulated with ET1 $(0.05 \mu \mathrm{M}, 48 \mathrm{~h})$ in the presence or absence of RR compound. C. BNP (Nppb) mRNA expression. D. Design of the GAPDH K225A conditional knock-in model. E. Heart tissue homogenates (after exposure to10-day TAC from WT or GAPDH K225A mutant) immunoprecipitated with Siah1 antibody and probed for GAPDH (left panel) and the quantification (right panel, results from 3 experiments). F. Echocardiogram: representative Mmode image (left), fractional shortening (FS) (right). n=4-7 in each group. Error bar represents mean \pm SEM. ${ }^{*} \mathrm{p}<0.05$ by unpaired two tailed $\mathrm{t}$ test; ${ }^{* * *} \mathrm{p}<0.001$ by one-way ANOVA with Tukey's multiple comparisons test. 
A

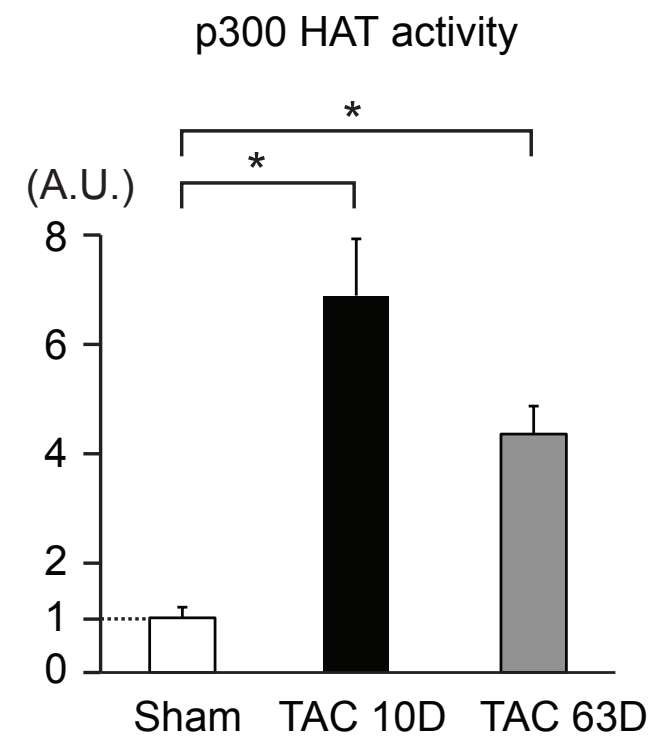

C
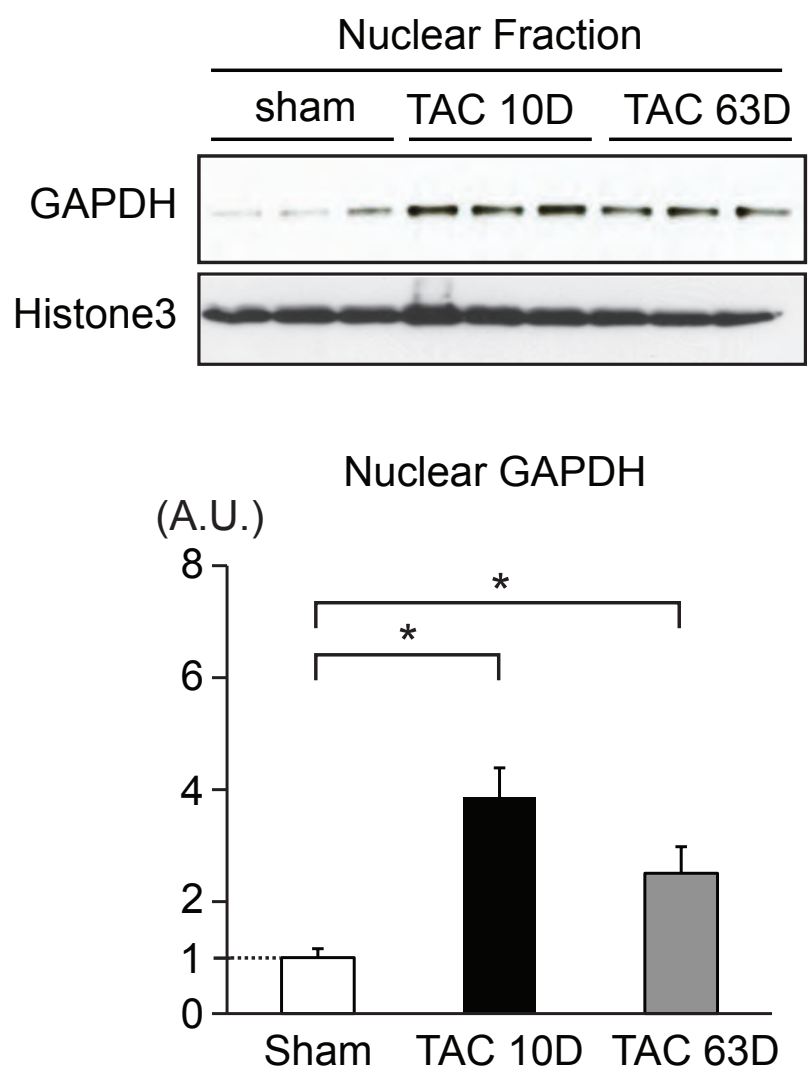
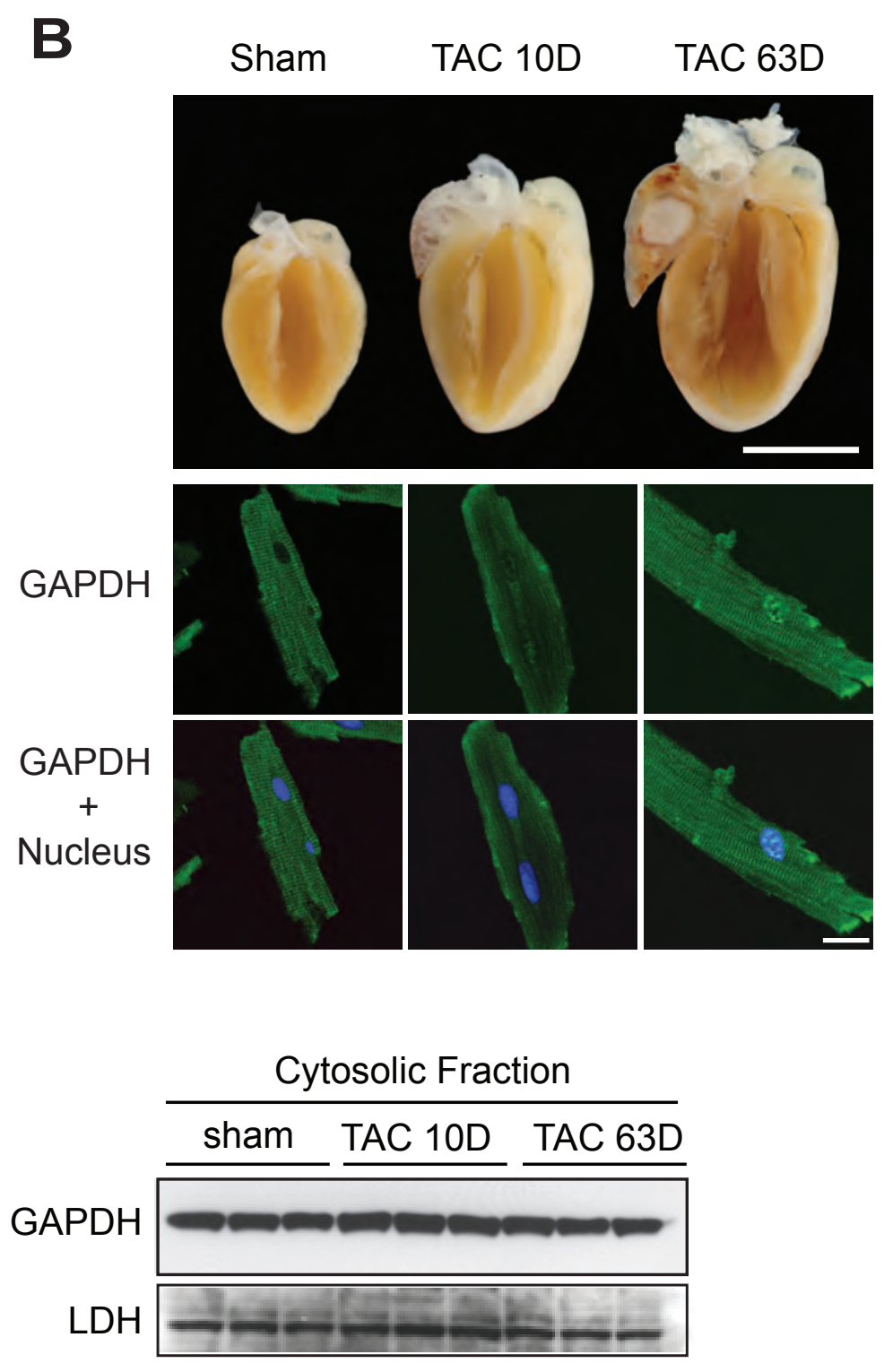

Cytosolic GAPDH

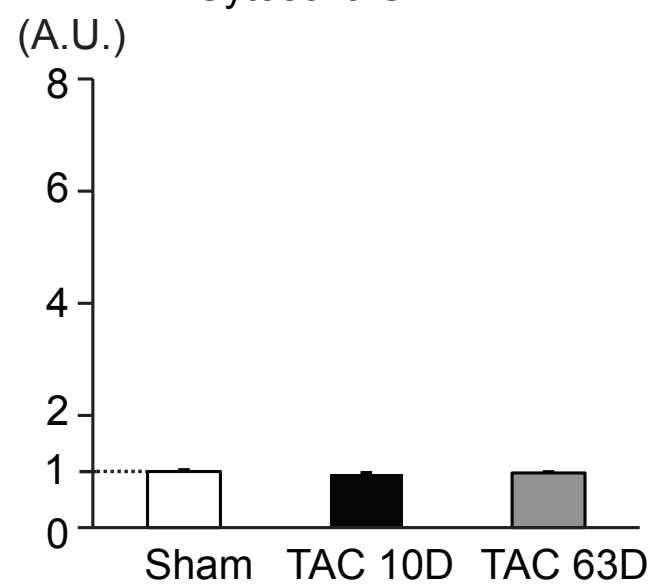


(1R, 3R)-1, 3-dimethyl-2-propargyl $-1,2,3,4$-tetrahydroisoquinoline (RR compound)<smiles>C#CCN1[C@@H](C)Cc2ccccc2[C@@H]1C</smiles>

B

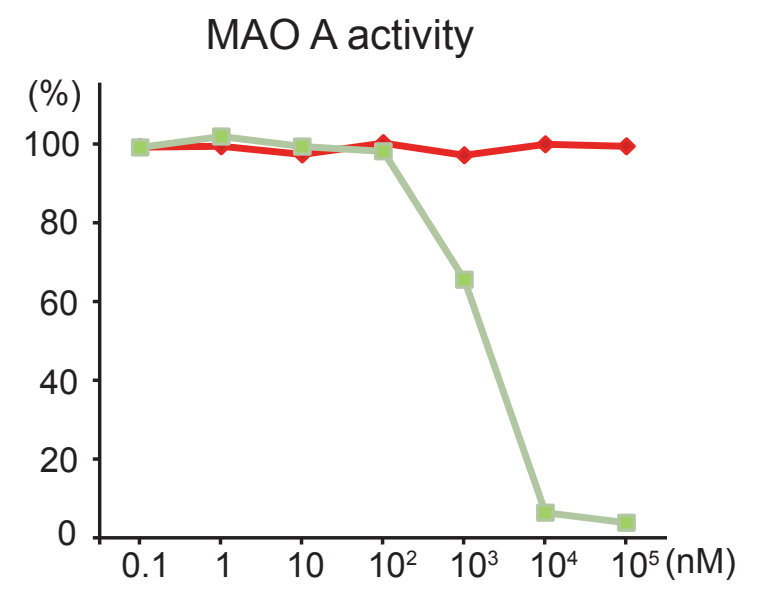

C

Glycolytic activity

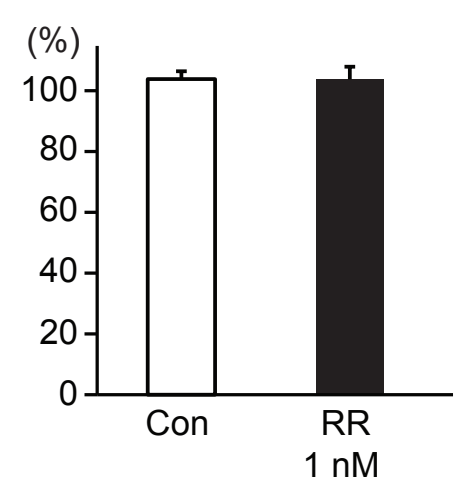

\section{Deprenyl (Dpr)}<smiles>C#CCN(C)[C@@H](C)Cc1ccccc1</smiles>

MAO B activity

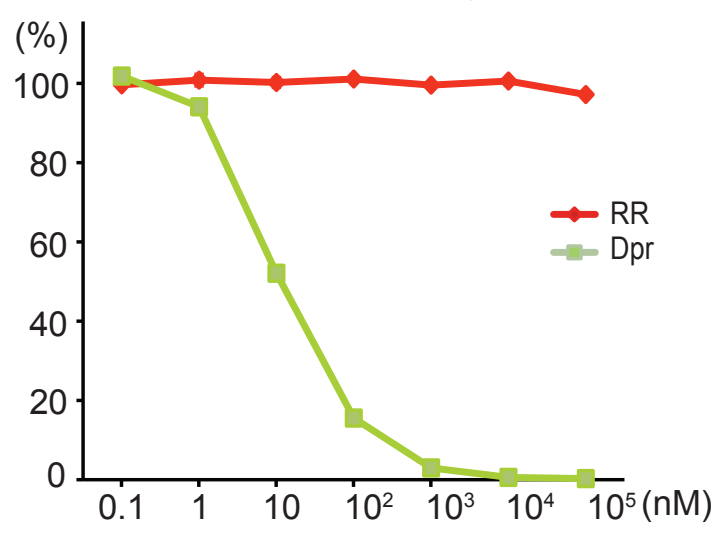



not certified by peer review) is the author/funder. All rights reserved. No reuse allowed without permission.

Heart weight / tibia length

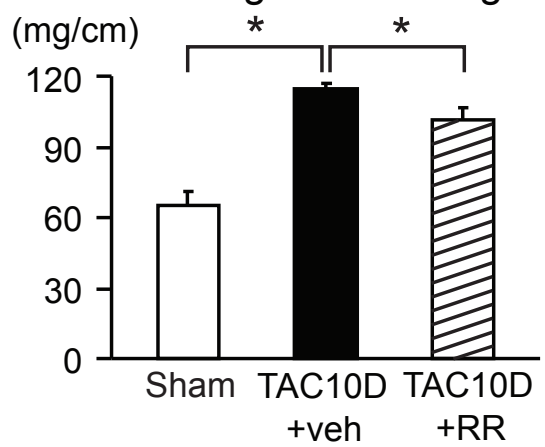

I

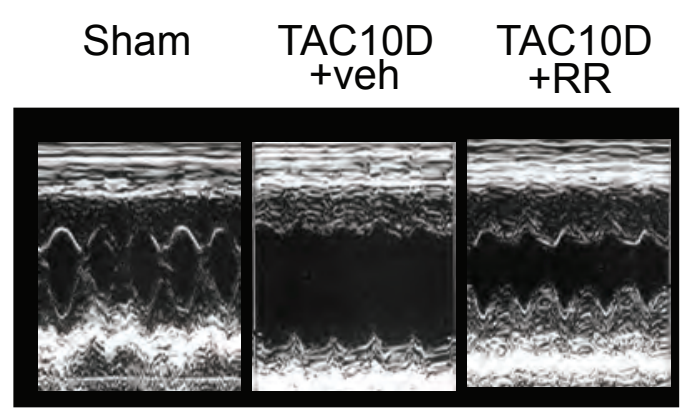

CSA of cardiomyocytes

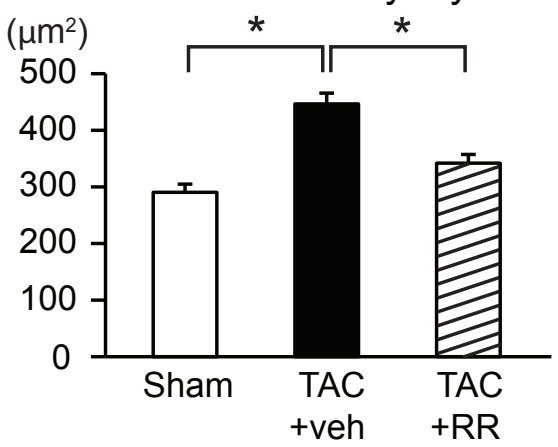

Fibrosis

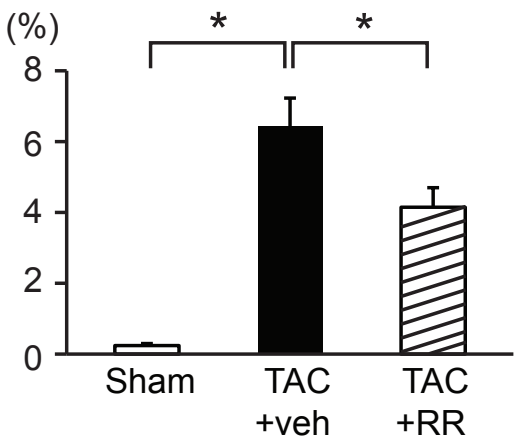

J
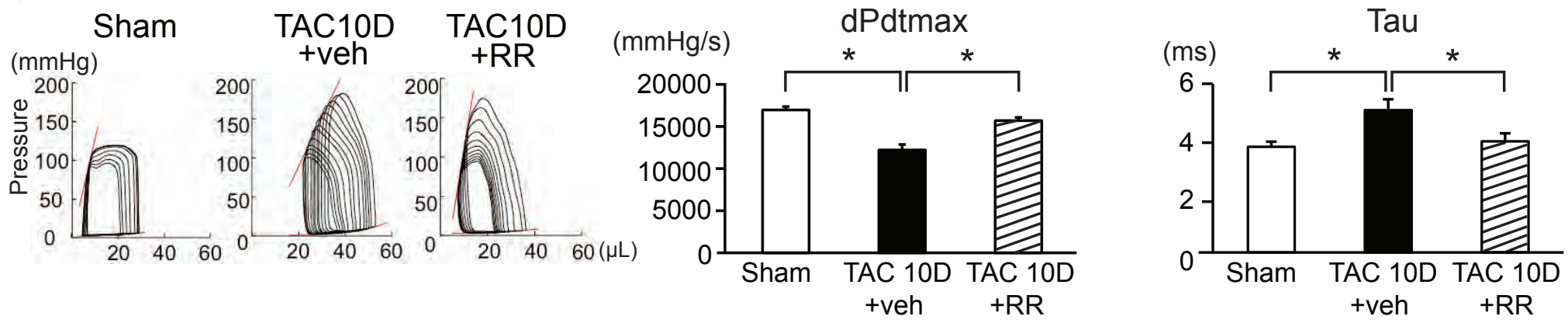

K

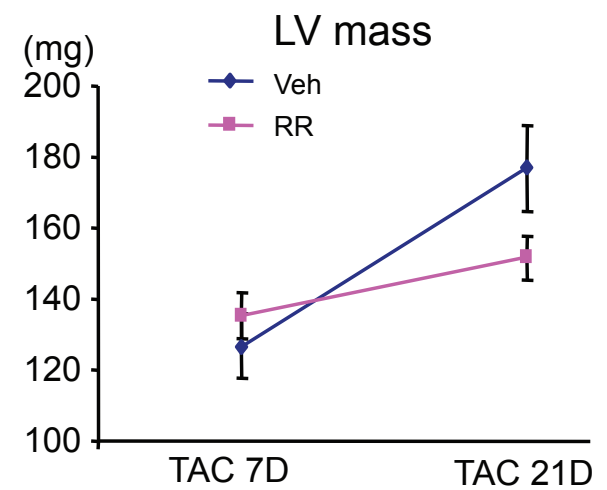

$\Delta \mathrm{LV}$ mass increase

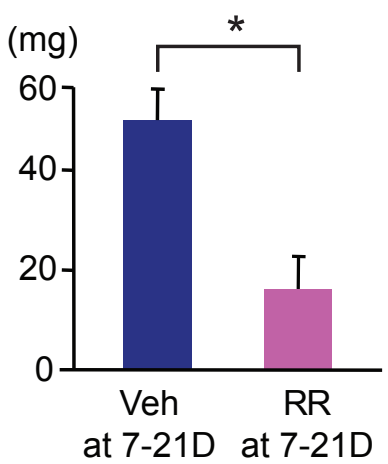

CSA of cardiomyocytes

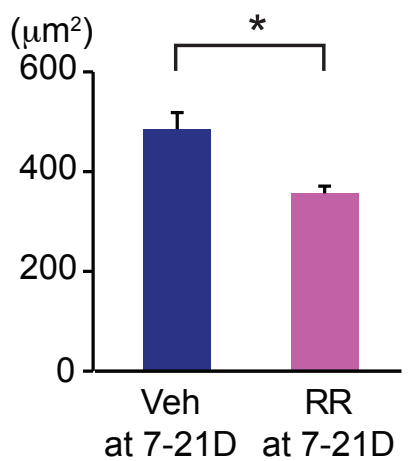


A

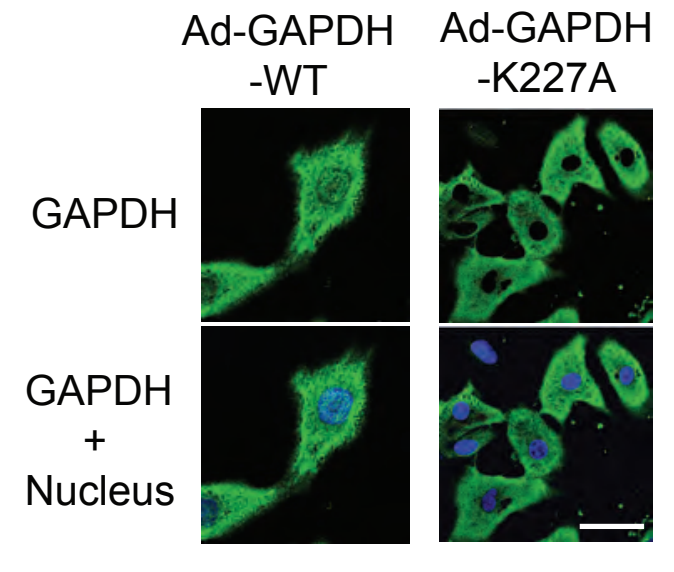

D

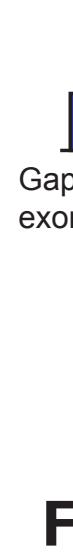

B

MEF2 activity

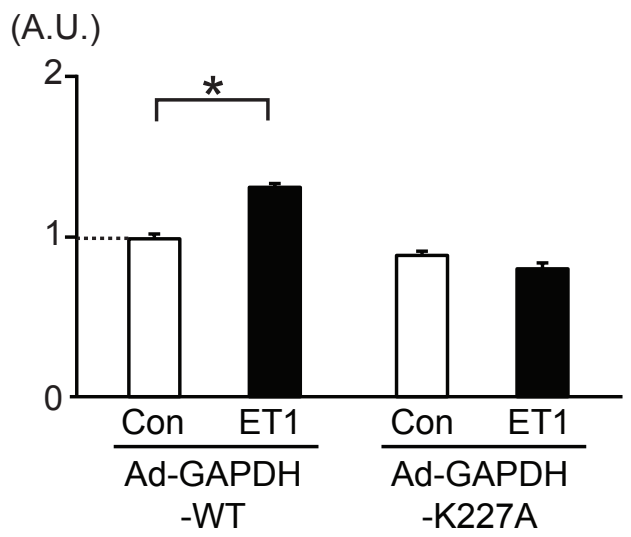

C

(A.U.)

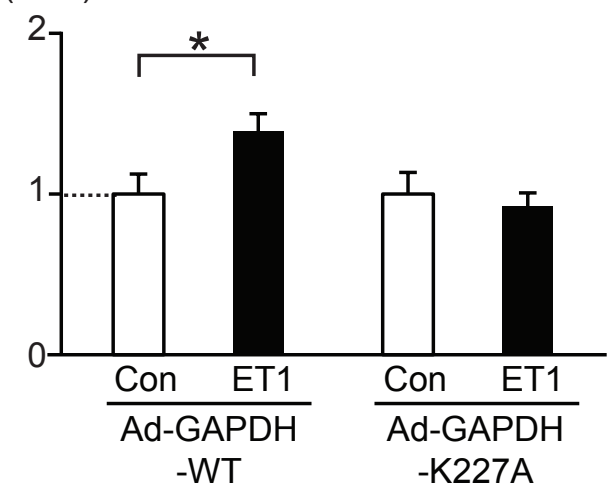

$\mathbf{E}$
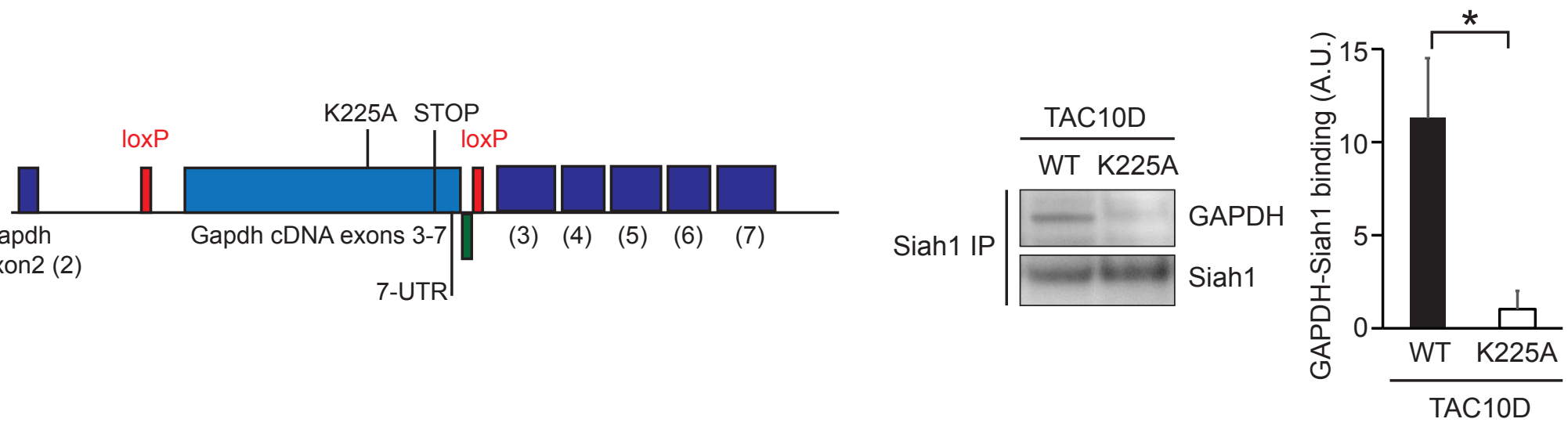

Sham

K225A

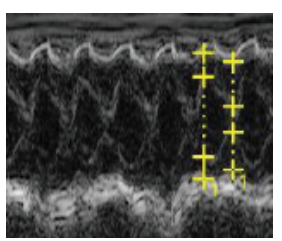

TAC10D

WT

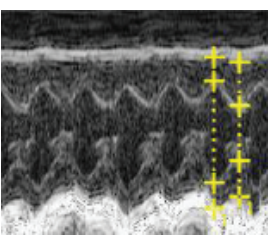

TAC10D

K225A

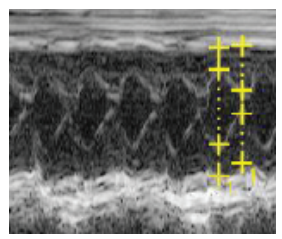

FS

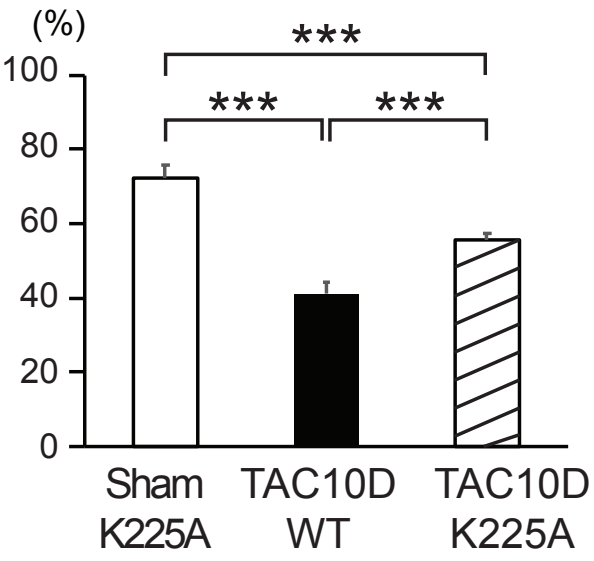




\section{Supplementary Materials:}

\section{Materials and Methods}

Reagents and RR compound: All reagents were purchased from Sigma, unless noted otherwise.

Details of the synthesis and characterization for RR are described in supplementary information.

In vitro screening for off-target activity of RR compound: HitProfilingScreen ${ }^{\circledR}$ based on radiolabeled binding assay was peformed by Eurofins Panlab Inc. to detect off-target activities of RR compound at the dose of $1.0 \mu \mathrm{M}$.

Animal models: All protocols were approved by the Animal Care and Use Committee of the Johns Hopkins University. TAC was performed in C57/BL6 mice (Jackson Laboratory) as previously described (1-3).

Physiological and histological analysis: Echocardiography and pressure-volume loop studies were performed as described previously (1-3). Heart samples fixed with $10 \%$ formalin were embedded in paraffin, sectioned and stained for myocyte size and fibrosis as described previously (2). Isolated myocytes were fixed with 50\% methanol/50\% acetone and stained with antibodies against GAPDH (Millipore), sulphonated GAPDH or HA as previously described (2, $3)$.

Rat neonatal cardiac myocyte culture: Rat neonatal cardiac myocytes were isolated from 1- to 2day-old Sprague-Dawley rats, and stimulated with $0.05 \mu \mathrm{M}$ ET-1 (1), with or without $1 \mathrm{nM}$ RR compound for $48 \mathrm{~h}$. Protein synthesis was assessed by $\left[{ }^{3} \mathrm{H}\right]$ leucine incorporation $(1,3)$. 
Adenoviral transfection of wild-type GAPDH and K227A mutant GAPDH was performed at 1030 moi as previously described (1).

Assays for HAT, MEF2, MAO, and GAPDH activity: p300 HAT activity was measured using a commercially available kit (Biovision), with immunoprecipitation with p300 antibody (4). MEF2 activity was measured by the luciferase reporter assay (Panomics). MAO activity assay was measured with a commercially available kit (Peninsula Laboratory). GAPDH activity was measured as previously described (5).

Protein and RNA analysis: Total RNA was isolated from cells or ventricular myocardium with Trizol and analyzed by real-time PCR with TaqMan probes (Applied Biosystems) (normalized to 18S RNA) (1-3). Cell lysates and nuclear extracts were obtained from cells or ventricular myocardium, and further analyzed by Western blotting as described (1-3).

Statistical analysis: Data are shown as means \pm SEM. Multiple group comparison was performed by one-way analysis of variance followed by the Bonferroni procedure for comparison of means. 


\section{Supplementary Text}

Synthesis and Characterization of $R R(6,7)$ : All solvents were reagent grade or HPLC grade. Unless otherwise noted, all materials were obtained from commercial suppliers and used without further purification. Melting points were taken on a Yanagimoto SP-M1 hot-stage melting point apparatus and are uncorrected. NMR spectra were measured using a JEOL JNM-AL300 (1H-NMR: $300 \mathrm{MHz},{ }_{13} \mathrm{C}-\mathrm{NMR}$ : $75 \mathrm{MHz}$ ) in $\mathrm{CDCl}_{3}$ with tetramethylsilane as an internal standard and the chemical shifts are given in $\delta$ values. High-resolution EIMS (HR-EIMS) was taken on a JEOL JMS-D300 mass spectrometer at $70 \mathrm{eV}$ (EIMS).

Elemental analysis was recorded on a Yanaco CHN-recorder MT-3. Optical rotations were determined using a JASCO DIP-1000 digital polarimeter in MeOH. CD spectra were measured on a JASCO J-600 spectrometer in MeOH. (1R,3R)-1,3-dimethyl-1,2,3,4tetrahydroisoquinoline was synthesized according to the literature methods1. $(1 R, 3 R)-1,3-$ dimethyl-1,2,3,4-tetrahydroisoquinoline was propargylated with propargyl bromide and cesium carbonate in a similar manner to the literature to afford a pale yellow oil2. $\mathrm{HCl}$ salt, colorless prisms, recrystallized from EtOH, mp $184^{\circ} \mathrm{C}$ (sublimation). ${ }_{1} \mathrm{H}-\mathrm{NMR}: 1.20(3 \mathrm{H}$, $\mathrm{d}, J=6.6 \mathrm{~Hz}), 1.44(3 \mathrm{H}, \mathrm{d}, J=6.8 \mathrm{~Hz}), 2.21(1 \mathrm{H}, \mathrm{t}, J=2.4 \mathrm{~Hz}), 2.57(1 \mathrm{H}, \mathrm{dd}, J=9.9,16.7$ Hz), $2.81(1 \mathrm{H}, \mathrm{dd}, J=4.7,16.7 \mathrm{~Hz}), 3.32(1 \mathrm{H}, \mathrm{dd}, J=2.4,16.6 \mathrm{~Hz}), 3.41-3.51(1 \mathrm{H}, \mathrm{m}), 3.55$ $(1 \mathrm{H}, \mathrm{dd}, J=2.4,16.6 \mathrm{~Hz}), 4.24(1 \mathrm{H}, \mathrm{q}, J=6.8 \mathrm{~Hz}), 7.03-7.16$ (4H, m). 13C-NMR: 17.7, 21.1, 34.3, 38.2, 47.4, 55.3, 72.0, 81.6, 125.8, 125.9, 127.3, 128.8, 133.4, 139.1. HREIMS m/z (M+): Calcd for $\mathrm{C}_{14} \mathrm{H}_{17} \mathrm{~N} ; 199.1358$ Found: 199.1358. Anal. Calcd for $\mathrm{C}_{14} \mathrm{H}_{18} \mathrm{ClN}$ (HCl salt): C, 71.32; H, 7.70; N, 5.94. Found: C, 71.08; H, 7.72; N, 5.88. $[\text { a }]_{\mathrm{D} 25.3}=+12.7(c=1.01 \%, \mathrm{MeOH}) . \mathrm{CD}\left(\mathrm{HCl}\right.$ salt, $\left.c=2.13 \times 10_{-3} \mathrm{M}, \mathrm{MeOH}\right)[\theta]_{25}(\mathrm{~nm})$ : +127 (271), -77 (267 valley), +123 (264), -13 (261, valley), +71 (257). 


\section{References}

1. E. Takimoto et al., Nat.Med. 11, 214 (2005).

2. M. Zhang et al., J. Am. Coll. Cardiol. 56, 2021 (2010).

3. E. Takimoto et al., J. Clin. Invest. 119, 408 (2009).

4. J. Q. Wei et al., Circulation 118, 934 (2008).

5. M. R. Hara et al., Nat. Cell Biol. 7, 665 (2005).

6. T. Saitoh, K. Shikiya, Y. Horiguchi, T. Sano, Chemical \& pharmaceutical bulletin 51, 667 (2003).

7. H. R. Tsou et al., Journal of medicinal chemistry 44, 2719 (2001). 


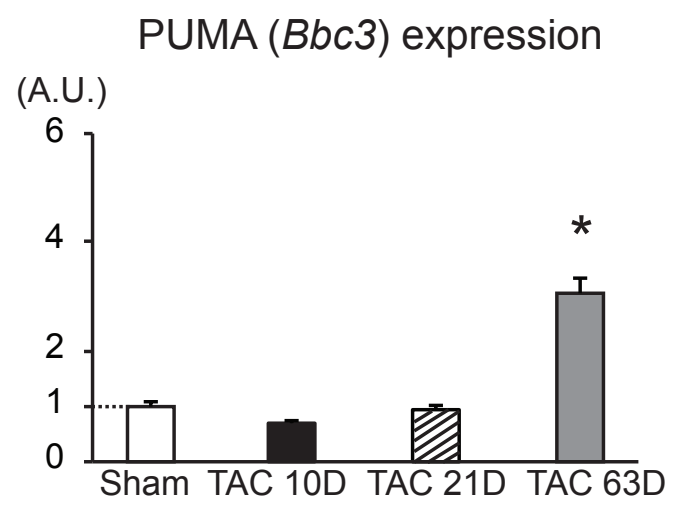

Fig. S1 
Fig. S1. No activation of the PUMA death cascade in the early stage TAC hearts.

PUMA mRNA $(B b c 3)$ expression in pressure-overloaded (TAC) hearts at different time points $(10,21$ and 63 days after TAC). Note that PUMA mRNA (Bbc3) expression was not increased in hearts after 10 days (TAC-10D) or 21 days (TAC-21D). $n=4$ in each group. ${ }^{*} \mathrm{p}<0.05$ vs all other groups. 
A

\section{nuclear GAPDH}

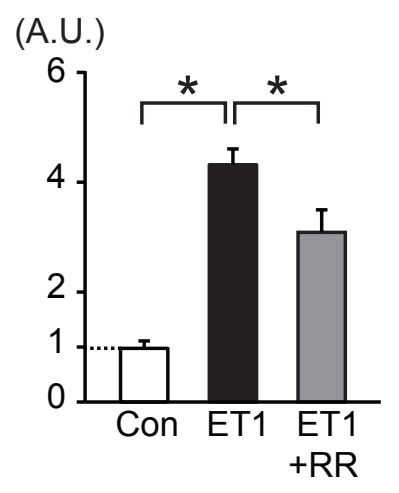

B

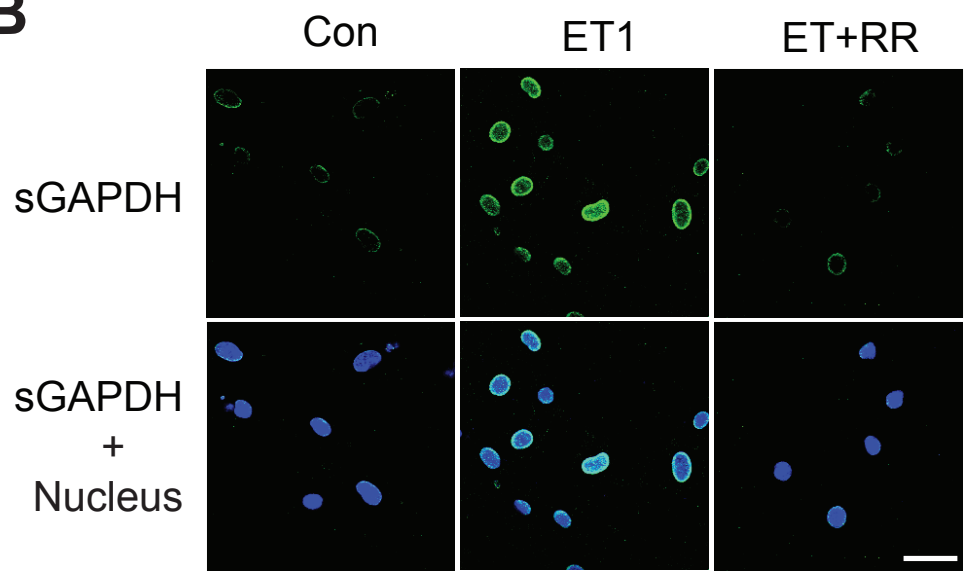

C

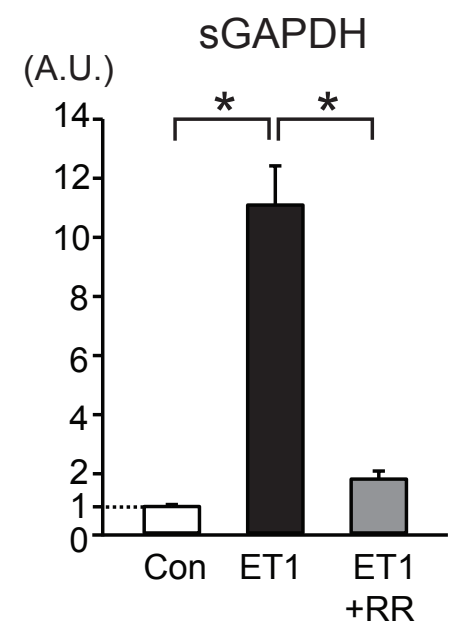

D

PUMA (Bbc3) expression

E
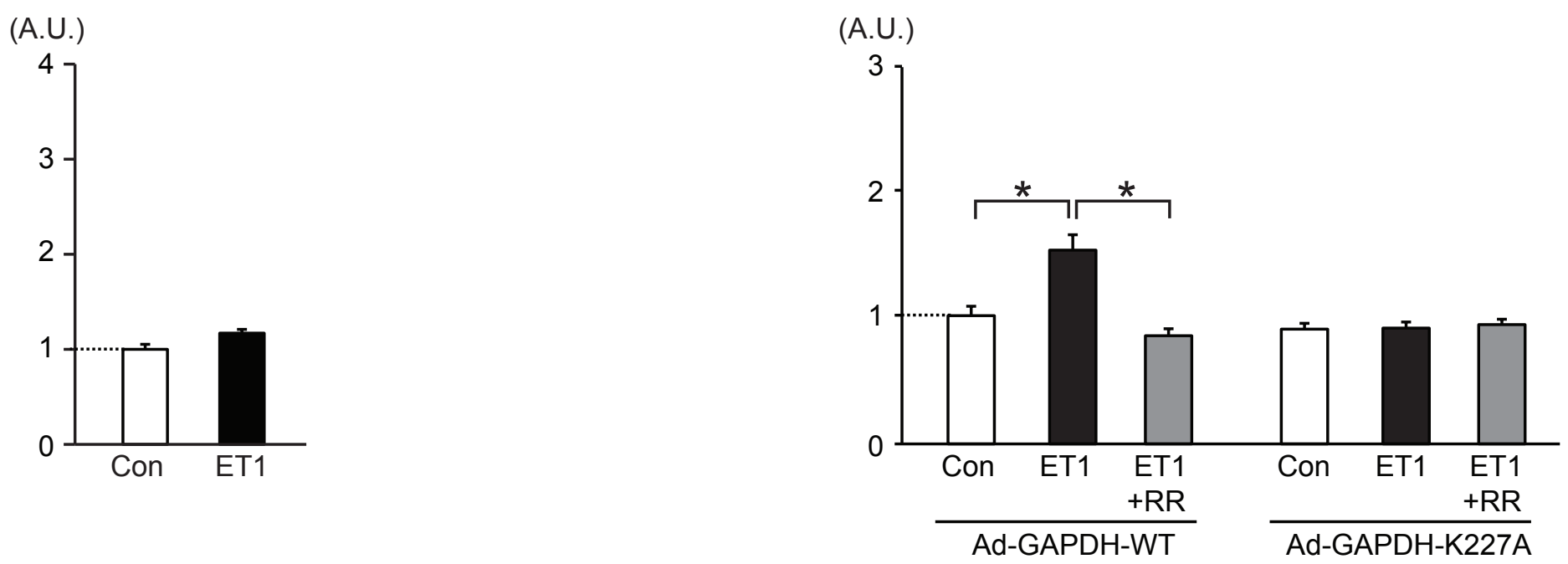

Fig. S2 
Fig. S2 Mechanisms of the nuclear GAPDH cascade in a cell model of heart hypertrophy.

A. Quantification of GAPDH staining (representative staining in Fig. 3B). Ratios of average intensity in the nucleus to that in cytosol for GAPDH are shown: 30 cells were analyzed in each group. B and C. Sulphonated (s) GAPDH staining of cultured rat cardiac myocytes exposed to ET1 with or without RR (B) and quantification (C). Scale bar, $50 \mu \mathrm{m}$. D. PUMA mRNA $(B b c 3)$ expression in cultured cardiac myocytes exposed to ET1 $(0.05 \mu \mathrm{M}, 48$ h). E. Cellular hypertrophy response to ET1 in the absence or presence of RR, assessed by cell surface area, in cells transfected with Ad-GAPDH-WT or with Ad-GAPDH-K227A. $* \mathrm{p}<0.05$ 
A

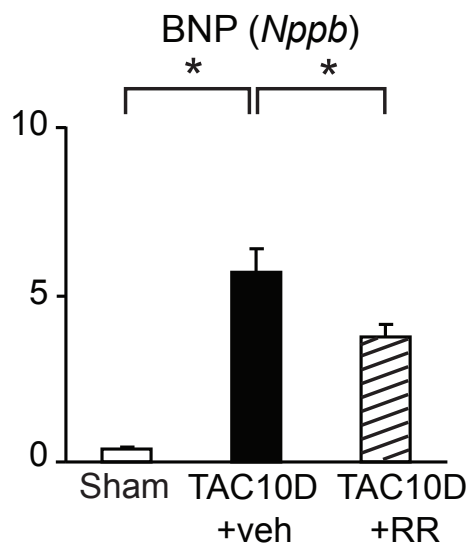

B

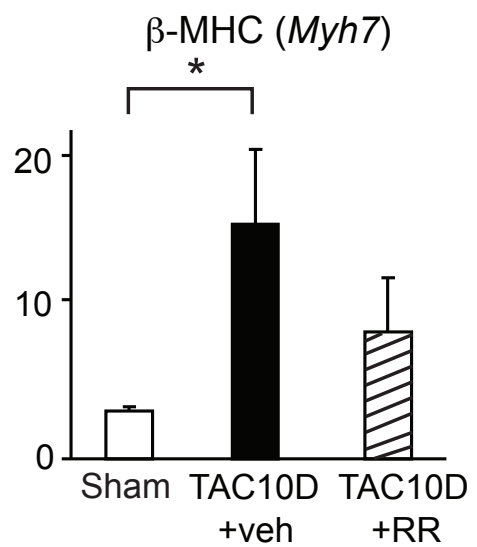

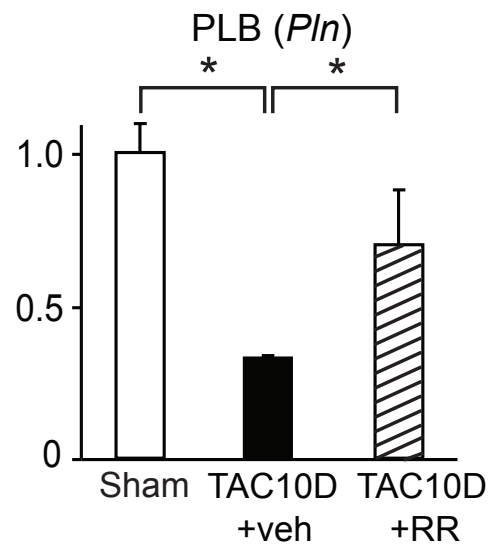

$\mathrm{EF}$
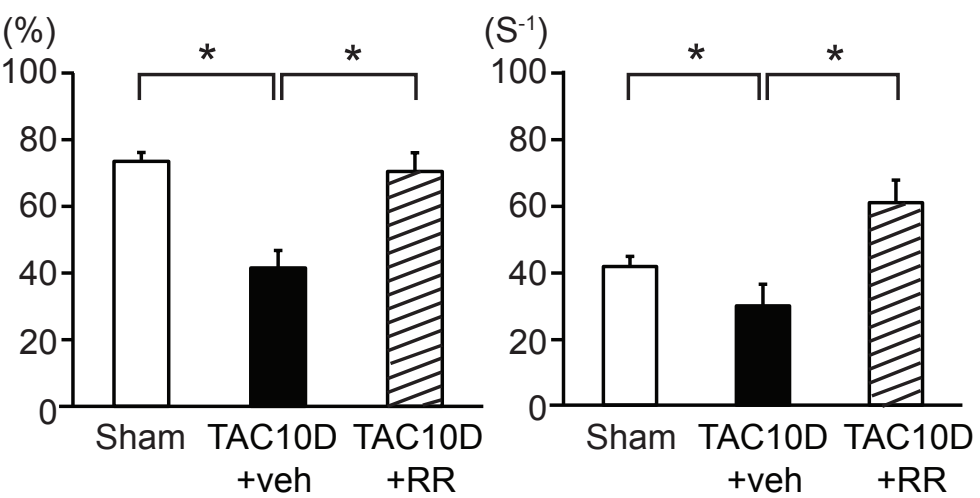

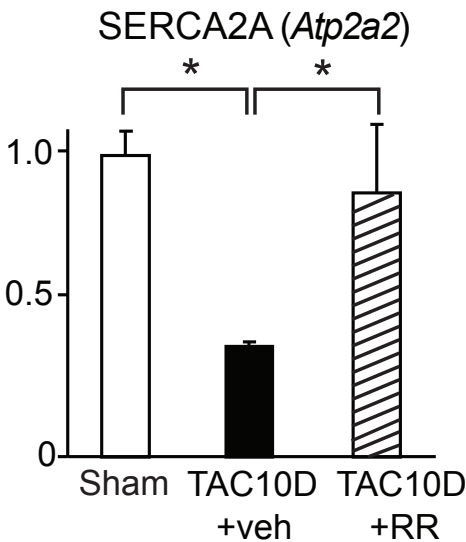

+veh $\quad+\mathrm{RR}$

D

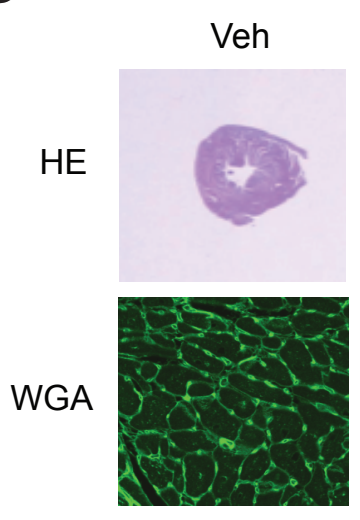

RR

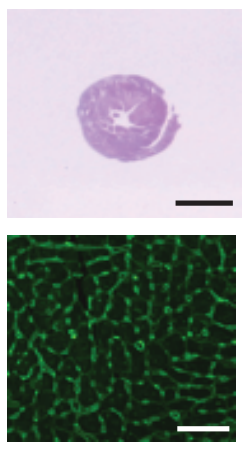

Peak LVP

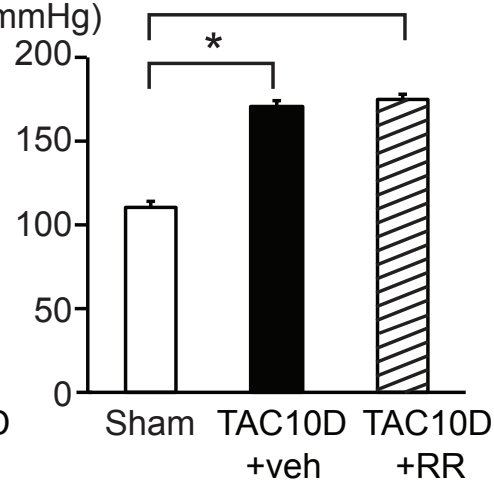

+veh

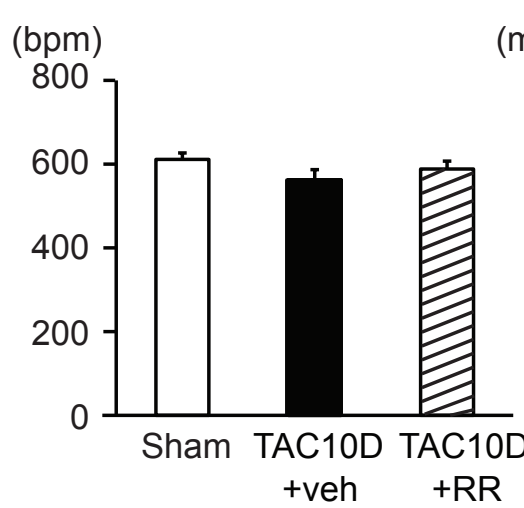

$\operatorname{BNP}(N p p b)$

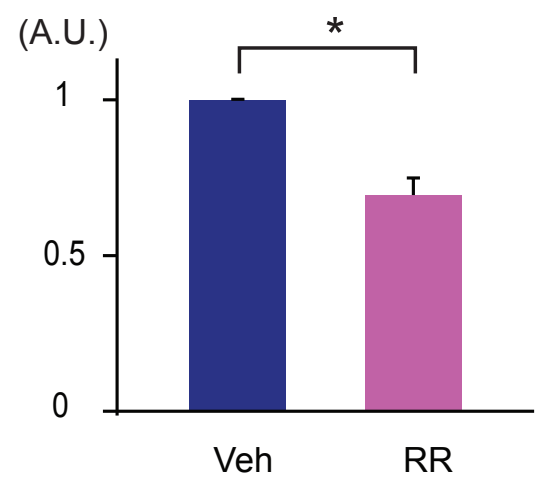


Fig. S3. Molecular, histological, and functional analyses of TAC hearts treated with the RR compound.

A and B. Myocardial mRNA expression levels of BNP (Nppb) and $\beta$-MHC $(M y h 7)(\mathrm{A})$ and calcium handling proteins $(A t p 2 a 2$ and $P \ln )(\mathrm{B}) . \mathrm{n}=4$ in each group. C. Hemodynamic parameters from pressure volume loop analysis, including heart rate, peak left ventricular pressure (LVP), ejection fraction (EF) and power max index (PMI). The latter two parameters reflect cardiac systolic function. Note that heart rate and afterload (peak LVP) were not affected by RR compound. $n=5-7$ in each group. D. Representative cross-section of hearts (left upper panels) and WGA staining (left lower panels) after 2 weeks of treatment with vehicle or RR to pre-existing hypertrophy, and myocardial mRNA expression of BNP (Nppb) (right bar graphs). Scale bars, $5 \mathrm{~mm}$ (upper panel) and $10 \mu \mathrm{m}$ (lower panel). $\mathrm{n}=4$ in each group. $* \mathrm{p}<0.05$. 


\section{FS at the baseline}

$(\%)$

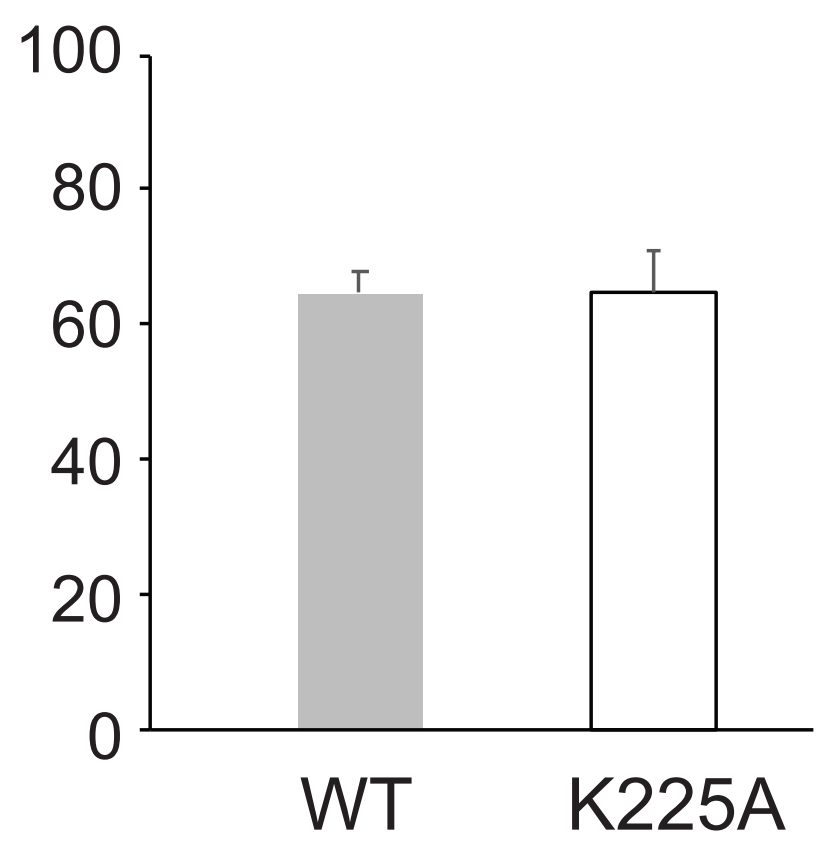

Fig. S4 
bioRxiv preprint doi: https://doi.org/10.1101/844902; this version posted November 16, 2019. The copyright holder for this preprint (which was not certified by peer review) is the author/funder. All rights reserved. No reuse allowed without permission.

Fig. S4. No difference in cardiac function at the baseline between wild type and K225A mutant hearts.

Cardiac function of wild types (WT) and K225A mutants at the baseline, assessed by echocardiographic measures of FS, were indistinguishable. 


\section{Table S1. In vitro screening for off-target activities of RR compound}

RR showed no significant interaction with primary targets of receptors, transporters, and enzymes in the Eurofins Panlabs Inc screen at the concentration of $1 \mu \mathrm{M}$, which is 1,000 fold high in comparison to that at which GAPDH-Siah1 binding was inhibited. 


\begin{tabular}{|c|c|}
\hline Receptor type & $\%$ inhibition \\
\hline Adenosine $A_{1}$ & 1 \\
\hline Adenosine $A_{2 A}$ & 8 \\
\hline Adrenergic $\alpha_{1 \mathrm{~A}}$ & 9 \\
\hline Adrenergic $\alpha_{1 B}$ & -3 \\
\hline Adrenergic $\alpha_{2 A}$ & 12 \\
\hline Adrenergic $\beta_{1}$ & 0 \\
\hline Adrenergic $\beta_{2}$ & 2 \\
\hline Calcium Channel L-Type, Dihydropyridine & -4 \\
\hline Cannabinoid $\mathrm{CB}_{1}$ & 9 \\
\hline Dopamine D1 & 4 \\
\hline Dopamine $\mathrm{D}_{2 \mathrm{~S}}$ & 4 \\
\hline GABAA, Flunitrazepam, Central & -3 \\
\hline GABAA, Muscimol, Central & 1 \\
\hline Glutamate, NMDA, Phencyclidine & 1 \\
\hline Histamine $\mathrm{H}_{1}$ & 0 \\
\hline Imidazoline I2, Central & 9 \\
\hline Muscarinic M2 & -2 \\
\hline Muscarinic $\mathrm{M}_{3}$ & 5 \\
\hline Nicotinic Acetylcholine & -3 \\
\hline Nicotinic Acetylcholine $\alpha 1$, Bungarotoxin & 3 \\
\hline Opiate $\mu(\mathrm{OP} 3, \mathrm{MOP})$ & 4 \\
\hline Phorbol Ester & 7 \\
\hline Potassium Channel [KaTP] & -10 \\
\hline Potassium Channel hERG & -2 \\
\hline Prostanoid $\mathrm{EP}_{4}$ & 3 \\
\hline Rolipram & 7 \\
\hline Serotonin (5-Hydroxytryptamine) 5- $\mathrm{HT}_{2 \mathrm{~B}}$ & 19 \\
\hline Sigma $\sigma 1$ & 7 \\
\hline Sodium Channel, Site 2 & 14 \\
\hline Transporter, Norepinephrine (NET) & 17 \\
\hline
\end{tabular}

